\title{
Pseudomonas aeruginosa quorum sensing molecules correlate with clinical status in cystic fibrosis
}

\author{
Helen L. Barr ${ }^{1}$, Nigel Halliday ${ }^{2}$, Miguel Cámara ${ }^{2}$, David A. Barrett ${ }^{3}$, \\ Paul Williams ${ }^{2}$, Douglas L. Forrester ${ }^{1}$, Rebecca Simms ${ }^{1}$, Alan R. Smyth ${ }^{4}$, \\ David Honeybourne ${ }^{5}$, Joanna L. Whitehouse ${ }^{5}$, Edward F. Nash ${ }^{5}$, Jane Dewar ${ }^{6}$, \\ Andrew Clayton ${ }^{6}$, Alan J. Knox ${ }^{1}$ and Andrew W. Fogarty ${ }^{7}$
}

\begin{abstract}
Affiliations: 'Division of Respiratory Medicine, Clinical Sciences Building, University of Nottingham, City Hospital Campus, Nottingham, UK. ${ }^{2}$ School of Life Sciences, Centre for Biomolecular Sciences, University of Nottingham, Nottingham, UK. ${ }^{3}$ Centre for Analytical Bioscience, School of Pharmacy, University of Nottingham, Nottingham, UK. "Division of Child Health, Obstetrics and Gynaecology, University of Nottingham, University Hospital, Nottingham, UK. ${ }^{5}$ West Midlands Adult CF Centre, Heart of England NHS Foundation Trust, Birmingham, UK. ${ }^{6}$ Dept of Respiratory Medicine, Nottingham University Hospitals NHS Trust, Nottingham, UK. ${ }^{7}$ Divison of Epidemiology and Public Health, Clinical Sciences Building, University of Nottingham, Nottingham, UK.
\end{abstract}

Correspondence: Helen L. Barr, Division of Respiratory Medicine, Clinical Sciences Building, University of Nottingham, City Hospital Campus, Nottingham, NG5 1PB, UK. E-mail: helen.barranottingham.ac.uk.

ABSTRACT Pseudomonas aeruginosa produces quorum sensing signal molecules that are potential biomarkers for infection.

A prospective study of 60 cystic fibrosis patients with chronic P. aeruginosa, who required intravenous antibiotics for pulmonary exacerbations, was undertaken. Clinical measurements and biological samples were obtained at the start and end of the treatment period. Additional data were available for 29 of these patients when they were clinically stable.

Cross-sectionally, quorum sensing signal molecules were detectable in the sputum, plasma and urine of $86 \%, 75 \%$ and $83 \%$ patients, respectively. They were positively correlated between the three biofluids. Positive correlations were observed for most quorum sensing signal molecules in sputum, plasma and urine, with quantitative measures of pulmonary $P$. aeruginosa load at the start of a pulmonary exacerbation. Plasma concentrations of 2-nonyl-4-hydroxy-quinoline (NHQ) were significantly higher at the start of a pulmonary exacerbation compared to clinical stability $(\mathrm{p}<0.01)$. Following the administration of systemic antibiotics, plasma 2-heptyl-4-hydroxyquinoline $(\mathrm{p}=0.02)$ and NHQ concentrations $(\mathrm{p}<0.01)$ decreased significantly.

In conclusion, quorum sensing signal molecules are detectable in cystic fibrosis patients with pulmonary $P$. aeruginosa infection and are positively correlated with quantitative measures of $P$. aeruginosa. NHQ correlates with clinical status and has potential as a novel biomarker for P. aeruginosa infection.

@ERSpublications

P. aeruginosa QS molecules correlate with clinical status in cystic fibrosis and are biomarkers for infection http://ow.ly/MhzZp

Received: Dec 082014 | Accepted after revision: April 082015 | First published online: June 112015

Clinical trial: This study is registered with at http://public.ukcrn.org.uk/search/ with identifier number 7026

This article has supplementary material available from erj.ersjournals.com

Support statement: This study was funded by the Medical Research Council grant number G0801558/1. Funding information for this article has been deposited with FundRef.

Conflict of interest: Disclosures can be found alongside the online version of this article at erj.ersjournals.com

Copyright OERS 2015. ERJ Open articles are open access and distributed under the terms of the Creative Commons Attribution Non-Commercial Licence 4.0. 


\section{Introduction}

The majority of adults with cystic fibrosis (CF) are chronically infected with Pseudomonas aeruginosa, which is associated with an accelerated decline in lung function [1], more frequent pulmonary exacerbations and increased mortality [2]. The success of $P$. aeruginosa, as an opportunistic pathogen, is partially attributed to the ability of whole bacterial populations of this bacterium to co-ordinate their activity using cell-to-cell communication, mediated by quorum sensing signal molecules (QSSMs) [3]. Quorum sensing (QS) regulates over $10 \%$ of the P. aeruginosa genome including swarming motility, biofilm maturation and antimicrobial resistance, as well as the production of virulence determinants such as elastases, pyocyanin, cyanide and exotoxins [3]. QS dependent virulence factors are elevated in bronchial secretions during episodes of pulmonary exacerbations in patients with CF [4]. These facts provide indirect evidence to suggest that QSSMs may contribute to changes in clinical status.

QSSMs are detectable in sputum obtained from patients with CF [5, 6] and QSSM concentrations in sputum are positively correlated with plasma levels [7]. This suggests that QSSMs have potential as biomarkers for $P$. aeruginosa in both pulmonary and non-pulmonary biological samples and could potentially be used to facilitate the diagnosis and response to treatment of this bacterium. The aim of this study is to test the hypothesis that QSSMs in sputum, plasma and urine correlate with clinical status in the adults with $\mathrm{CF}$ at the beginning and end of a pulmonary exacerbation, and also compared to clinical stability. Our data show that systemic concentrations of several QSSMs correlate with changes in clinical status, suggesting that they have potential as novel biomarkers for airway infection with P. aeruginosa.

\section{Methods}

\section{Participants}

Participants were recruited from two UK adult CF specialist centres, Nottingham University Hospitals NHS Trust, Nottingham and Heart of England NHS Foundation Trust, Birmingham. Inclusion criteria were a diagnosis of $\mathrm{CF}$, age 16-60 years and chronic pulmonary infection with $P$. aeruginosa (defined as $>50 \%$ of cultures positive for $P$. aeruginosa in $\leq 12$ months). Patients with chronic $P$. aeruginosa and co-infections with other organisms were included in the study. The duration of chronic pulmonary infection was defined as the earliest known date that $P$. aeruginosa was isolated that led to $>50 \%$ positive sputum cultures in the subsequent 12 months. Exclusion criteria were any previous isolation of Burkholderia cepacia complex in sputum, previous lung transplant, pregnancy or advanced disease. The local ethics committee approved the study (Nottingham Research Ethics Committee 1 ref. 09/H0407/1) and the study was registered with the UK clinical research network (http://public.ukcrn.org.uk/search/ with identifier number 7206). Informed written consent was obtained for all participants.

\section{Study design}

This was a prospective observational study of patients with CF undergoing standard intravenous treatment for a pulmonary exacerbation, defined using the Rosenfeld criteria [8]. Both the antibiotic therapy and the duration of treatment were chosen at the discretion of the patient's clinician, and treatment decisions were based on current clinical guidelines [9]. Spontaneous sputum, $8 \mathrm{~mL}$ blood and $25 \mathrm{~mL}$ urine samples were obtained within $72 \mathrm{~h}$ of the start and end of $i . v$. antibiotic therapy. Pulmonary function tests were performed according to standardised criteria [10] at the start and end of i.v. therapy. Comparable paired samples were also collected at clinical stability for a subset of patients, either before or after the exacerbation of interest, when the participant had not experienced a pulmonary exacerbation requiring i.v. antibiotics in the previous 4 weeks. Patients were recruited only once into the study. CF related diabetes mellitus was defined according to national guidelines [11]. Sputum or cough swabs were processed in the hospital laboratory using standard microbiological techniques [12].

\section{Sample analysis}

Sputum plugs were harvested and processed for differential cell analysis [13], quantitative microbiology, measurement of QSSMs [14, 15] and routine hospital microbiological culture [12]. Sputum plugs for quantitative microbiological analysis were mixed with an equal volume of dithiothreitol and diluted with $0.9 \%$ weight/volume saline and $100 \mu \mathrm{L}$ of $\times 10^{-2}$ and $\times 10^{-4}$ solutions were plated on blood and Pseudomonas isolation agar (Difco; BD, Sparks, MD, USA). The plates were incubated at $37^{\circ} \mathrm{C}$ and colony counts were performed daily between $24 \mathrm{~h}$ and $72 \mathrm{~h}$, until maximal growth was achieved.

\section{QSSM analysis}

Sputum samples for QSSM analysis were extracted using acidified ethyl acetate (Fisher Chemicals, Loughborough, UK) $[14,15]$. Urine and plasma samples were extracted by solid phase extraction (further details are provided in the online supplementary material). Prepared samples were then analysed by liquid 
chromatography-tandem mass spectrometry (LC-MS/MS), further details are provided in the online supplementary material [14].

\section{Statistics}

Spearman's rank correlations and Wilcoxon matched pairs signed-rank tests were used appropriately (further details are provided in the online supplementary material).

\section{Results}

\section{Study population}

60 participants were enrolled (50 patients from Nottingham University Hospitals NHS Trust and 10 patients from Heart of England NHS Foundation Trust) of which 34 (57\%) were male. One participant died during the hospital admission and data were only available for this patient at the start of the exacerbation. Additional samples were collected from 29 participants at clinical stability, with eight samples taken before the exacerbation of interest and 21 samples post exacerbation.

The i.v. therapy consisted of at least two antipseudomonal antibiotics, usually an aminoglycoside combined with an antipseudomonal $\beta$-lactam (table 1) for a median (range) of 14 days (9-29 days). The median (IQR) for the duration of pulmonary infection with $P$. aeruginosa was 7.6 years (3.7-12.5). 55 (92\%) patients were

TABLE 1 Participants' characteristics, sputum microbiology and antibiotics administered at baseline

\begin{tabular}{|c|c|}
\hline \multicolumn{2}{|l|}{ Demographics } \\
\hline Age years & $29.3 \pm 10.4$ \\
\hline Height $\mathrm{m}$ & $1.68 \pm 0.09$ \\
\hline Body mass index $\mathrm{kg} \cdot \mathrm{m}^{-2}$ & $21.9 \pm 3.7$ \\
\hline \multicolumn{2}{|l|}{ Spirometry\# } \\
\hline Absolute FEV1 L & $1.70 \pm 0.70$ \\
\hline FEV $1 \%$ pred & $47.2 \pm 16.9$ \\
\hline Absolute forced vital capacity L & $2.79 \pm 1.03$ \\
\hline Forced vital capacity \% pred & $65.4 \pm 19.2$ \\
\hline \multicolumn{2}{|l|}{ Maintenance therapies } \\
\hline Nebulised colomycin & $38(63)$ \\
\hline Nebulised tobramycin & $6(10)$ \\
\hline Nebulised DNAse & $36(60)$ \\
\hline Nebulised hypertonic saline & $21(35)$ \\
\hline Oral corticosteroids & $11(18)$ \\
\hline Oral azithromycin & $49(82)$ \\
\hline \multicolumn{2}{|l|}{ Hospital microbiology results } \\
\hline Pseudomonas aeruginosa alone & $42(70)$ \\
\hline \multicolumn{2}{|l|}{ Pseudomonas aeruginosa co-infection } \\
\hline MSSA & $5(8)$ \\
\hline MRSA and Haemophilus influenzae & $1(2)$ \\
\hline MRSA & $1(2)$ \\
\hline Candida albicans & $4(7)$ \\
\hline \multicolumn{2}{|l|}{ Pseudomonas aeruginosa not isolated } \\
\hline MRSA alone & $2(3)$ \\
\hline MSSA alone & $1(2)$ \\
\hline MSSA and Aspergillus fumigatus & $1(2)$ \\
\hline Respiratory commensals only & $3(5)$ \\
\hline \multicolumn{2}{|l|}{ Intravenous antibiotic administered } \\
\hline Tobramycin & $32(53)$ \\
\hline Ceftazidime & $29(48)$ \\
\hline Colomycin & $24(40)$ \\
\hline Meropenem & $24(40)$ \\
\hline Aztreonam & $7(12)$ \\
\hline Piperacillin/tazobactam & $5(8)$ \\
\hline Other & $5(8)$ \\
\hline
\end{tabular}

Data are presented as mean \pm SD or $n(\%)$. FEV 1 : forced expiratory volume in $1 \mathrm{~s}$; MSSA: methicillin-sensitive Staphylococcus aureus; MRSA: methicillin-resistant S. aureus. \#: $n=58$; ๆ: other antibiotics consisted of intravenous flucloxacillin ( $n=2)$, septrin $(n=1)$, linezolid $(n=1)$ and ofloxacillin $(n=1)$. 
pancreatic exocrine insufficient, 33 (55\%) patients had a diagnosis of CF related diabetes mellitus, 13 (22\%) patients had CF related liver disease, 8 (13\%) patients had allergic bronchopulmonary aspergillosis and $2(3 \%)$ had asthma. 57 (95\%) participants produced a sputum sample for hospital microbiological analysis and three required a cough swab. P. aeruginosa was isolated from $53(88 \%)$ samples, of which 42 (70\%) samples had $P$. aeruginosa only isolated from them and $11(18 \%)$ samples had $P$. aeruginosa isolated in combination with other microorganisms (table 1).

\section{Individual QSSMs in sputum, plasma and urine were significantly correlated with each other at the start of a pulmonary exacerbation}

Cross-sectionally, at least one QSSM was detectable in the sputum, plasma and urine of 51 (86\%), 42 (75\%) and $49(83 \%)$, respectively, of patients who provided samples at the start of a pulmonary exacerbation. Eight different QSSMs were detectable in sputum, six were detectable in plasma and four were detectable in urine (table 2). 2-nonyl-3-hydroxy-4(1H)-quinolone (C9-PQS), was only detected in the plasma of one patient and was not included in further analysis. Detection rates for QSSMs at the start of antibiotics in each biofluid ranged from $8(14 \%)$ for plasma 2-heptyl-3-hydroxy-4(1H)-quinolone (PQS) to 48 (81\%) for urinary 2-heptyl-4-hydroxyquinoline- $N$-oxide (HQNO) (table 2). Of the 53 patients with positive $P$. aeruginosa culture from the hospital laboratory at baseline, QSSMs were detectable in the sputum, plasma and urine of 47 (89\%), 39 (78\%) and 44 (85\%) samples respectively. Of the seven patients whose sputum did not isolate P. aeruginosa in the hospital laboratory at baseline, QSSMs were detected in all three biofluids from two patients, in the sputum and urine from two patients, in the urine and plasma of one patient and none were detected in any of the biofluids tested from two patients (table S1). Subsequent hospital laboratory sputum cultures isolated $P$. aeruginosa in all seven cases with a median (IQR) of 4.0 months (1.5-6.9 months) post hospitalisation. There were no significant associations observed between prior maintenance usage of azithromycin and QSSM levels in any of the biofluids.

2-heptyl-4-hydroxyquinoline (HHQ), 2-nonyl-4-hydroxyquinoline (NHQ), HQNO and 2-nonyl-4hydroxyquinoline- $N$-oxide (NQNO) had clearly identifiable peaks in all three body fluids allowing

TABLE 2 Quorum sensing molecule signal concentrations in sputum, plasma and urine before and after administration of intravenous antibiotics for a pulmonary exacerbation in cystic fibrosis patients

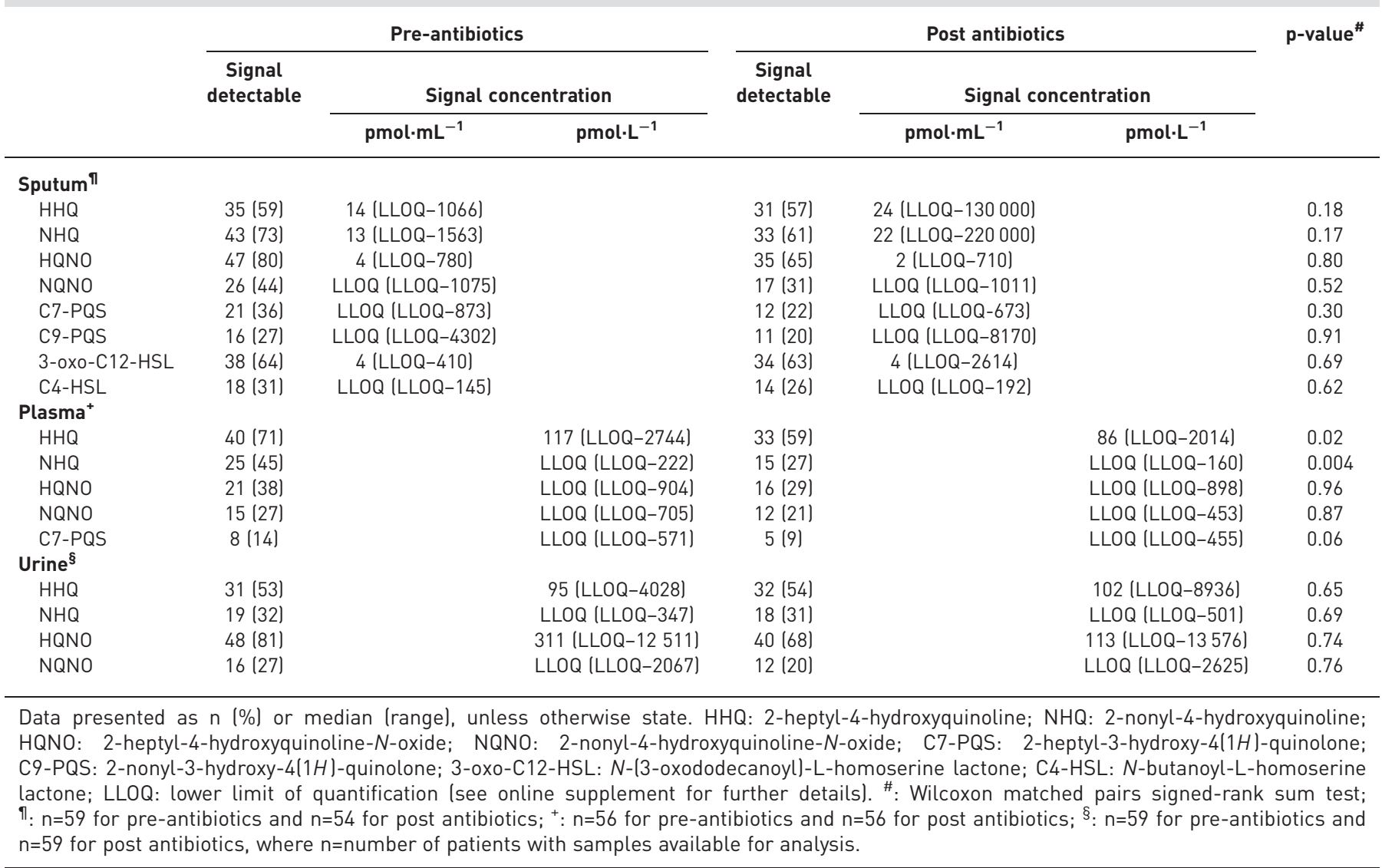


TABLE 3 Cross-sectional association between baseline sputum, plasma and urinary signal concentrations for the four alkyl quinolones detected in all three media

\begin{tabular}{lccc} 
QSSM & Sputum and plasma\# & Sputum and urine & Plasma and urine $^{+}$ \\
\hline HHQ & $0.72(<0.0001)$ & $0.63(<0.0001)$ & $0.80(<0.0001)$ \\
NHQ & $0.49(0.0002)$ & $0.65(<0.0001)$ & $0.54(<0.0001)$ \\
HQNO & $0.56(<0.0001)$ & $0.61(<0.0001)$ & $0.63(<0.0001)$ \\
NQNO & $0.58(<0.0001)$ & $0.13(0.34)$ & $0.32(0.02)$
\end{tabular}

Data are presented as Spearman's correlation coefficient ( $p$-value). QSSM: quorum sensing signal molecule; HHQ: 2-heptyl-4-hydroxyquinoline; NHQ: 2-nonyl-4-hydroxyquinoline; HQNO: 2-heptyl-4-hydroxyquinoline-N-oxide; NQNO: 2-nonyl-4-hydroxyquinoline-N-oxide. ${ }^{\#}: n=55 ; ~^{\eta}: n=58 ;{ }^{+}: n=56$, where $n$ is number of participants with samples available for analysis.

comparisons between the levels of these molecules in different biofluids. At the start of the pulmonary exacerbation, sputum, plasma and urinary levels of HHQ, NHQ and HQNO were positively correlated with each other in all three biofluids (table 3). Overall, the strongest relationships were found for HHQ, with the sputum signal positively correlated with both plasma $(\mathrm{r}=0.72, \mathrm{p}<0.0001)$ and urinary concentrations $(\mathrm{r}=0.63, \mathrm{p}<0.0001)$. For all four molecules, plasma levels were significantly correlated with both sputum $(\mathrm{p}<0.001$ for all comparisons) and urinary levels ( $\mathrm{p}=0.02$ to $<0.001)$.

QSSMs were positively associated with higher quantitative load of $P$. aeruginosa at the start of a pulmonary exacerbation

A higher $P$. aeruginosa load, at the start of antibiotic treatment, was associated with increased signal concentration in six of the eight QSSMs detected in sputum (table 4) with the highest correlation demonstrated with $N$-butanoyl-L-homoserine lactone $(\mathrm{r}=0.53, \mathrm{p}<0.0001)$. At the start of antibiotic treatment, all plasma QSSMs were associated with $P$. aeruginosa density; the strongest relationship between bacterial

\begin{tabular}{|c|c|c|}
\hline QSSM & Spearman's correlation coefficient & p-value \\
\hline \multicolumn{3}{|l|}{ Sputum $\#$} \\
\hline$H H Q$ & 0.52 & 0.0001 \\
\hline $\mathrm{NHQ}$ & 0.50 & 0.0001 \\
\hline HQNO & 0.35 & $<0.01$ \\
\hline NQNO & 0.34 & 0.01 \\
\hline C7-PQS & 0.43 & 0.001 \\
\hline C9-PQS & 0.27 & $<0.05$ \\
\hline 3-oxo-C12-HSL & 0.27 & $<0.05$ \\
\hline C4-HSL & 0.54 & $<0.0001$ \\
\hline \multicolumn{3}{|l|}{ Plasma } \\
\hline$H H Q$ & 0.51 & $<0.001$ \\
\hline $\mathrm{NHQ}$ & 0.30 & 0.03 \\
\hline HQNO & 0.49 & $<0.001$ \\
\hline NQNO & 0.35 & 0.01 \\
\hline C7-PQS & 0.37 & $<0.01$ \\
\hline \multicolumn{3}{|l|}{ Urine $^{+}$} \\
\hline$H H Q$ & 0.30 & 0.03 \\
\hline $\mathrm{NHQ}$ & 0.29 & 0.03 \\
\hline HQNO & 0.32 & 0.02 \\
\hline NQNO & 0.36 & $<0.01$ \\
\hline
\end{tabular}

Median (interquartile rangel for sputum bacterial load of Pseudomonas aeruginosa was $9.6 \times 10^{6} \mathrm{CFU} \cdot \mathrm{g}^{-1}$ $\left(1.3 \times 10^{6}-5.7 \times 10^{7} \mathrm{CFU} \cdot \mathrm{g}^{-1}\right)$. HHQ: 2-heptyl-4-hydroxyquinoline; NHQ: 2-nonyl-4-hydroxyquinoline; HQNO: 2-heptyl4-hydroxyquinoline- $N$-oxide; NQNO: 2-nonyl-4-hydroxyquinoline- $N$-oxide; C7-PQS: 2-heptyl-3-hydroxy-4(1H)-quinolone; C7-PQS: 2-nonyl-3-hydroxy-4(1H)-quinolone; 3-oxo-C12-HSL: $N$-(3-oxododecanoyl)-L-homoserine lactone; C4-HSL: N-butanoyl-L-homoserine lactone. ${ }^{\#}: \mathrm{n}=54 ;{ }^{\natural}: \mathrm{n}=50 ;{ }^{+}: \mathrm{n}=53$, where $\mathrm{n}$ is the number of patients with samples available for analysis. 
load and QSSM was demonstrated for HHQ $(r=0.51, p<0.001)$. Similarly, at the start of antibiotic treatment all urine levels of QSSMs were also associated with quantitative measures of $P$. aeruginosa load; the strongest association observed was for NQNO $(r=0.36, \mathrm{p}<0.01)$. Significantly positive associations were found between per cent predicted forced expiratory volume in $1 \mathrm{~s}(\mathrm{FEV} 1)$ and sputum HHQ ( $\mathrm{r}=0.31, \mathrm{p}=0.02)$ (fig. S1) and NHQ ( $r=0.30, p=0.02$ ) (fig. S2), but not with other QSSMs in any media. No significant associations were found between sputum neutrophil concentrations and QSSM levels (data not shown).

\section{Longitudinal analysis at start and end of exacerbation: plasma $H H Q$ and $N H Q$ concentrations fell significantly after i.v. antibiotics}

Following the administration of antibiotics, plasma concentrations of HHQ and NHQ decreased significantly (table 2). For HHQ, the number of samples with detectable signal fell from 40 (71\%) to 33 (59\%) after the administration of antibiotics $(\mathrm{p}=0.02)$ and the comparable figures for NHQ were a decrease from $25(45 \%)$ samples with detectable signal to $15(27 \%)$ positive samples $(\mathrm{p}=0.004)$. However, corresponding sputum and urinary signals did not change significantly for either QSSM (table 2). No changes in QSSM signal concentration were demonstrated for the remaining QSSMs detected in sputum, plasma or urine following treatment with antibiotics (table 2). Over the same period, mean (95\% CI) increase in FEV1 was $253 \mathrm{~mL}$ $(179-326 \mathrm{~mL})$ or $6.1 \%$ pred $(4.0-8.1 \%$ pred) and the increase in FVC was $269 \mathrm{~mL}(145-393 \mathrm{~mL})$ or $6.3 \%$ pred $\left(3.3-9.2 \%\right.$ pred). Mean sputum neutrophil concentration decreased from 7.1 to $6.9 \log \mathrm{cells}^{-\mathrm{g}^{-1}}$ $(\mathrm{p}<0.001)$ following antibiotic therapy, but no change in quantitative microbiological load was demonstrated using blood $(\mathrm{p}=0.22)$ (table S2) or pseudomonas isolation agar $(\mathrm{p}=0.98)$ (table S2).

\section{Longitudinal analysis from the start of the exacerbation compared with clinical stability: plasma NHQ increased at presentation with exacerbation}

Data were available from 29 individuals who provided paired data at the beginning of the clinical exacerbation and also during a period of clinical stability. Compared to the period of clinical stability, plasma NHQ was increased at presentation for treatment at the beginning of an exacerbation $(p=0.008)$ (fig. 1), while sputum NQNO, sputum C7-PQS and plasma NQNO were lower at this time ( $\mathrm{p}=0.009$, $\mathrm{p}=0.03$ and $\mathrm{p}=0.003$, respectively). There was no difference in quantitative microbiological load of $P$. aeruginosa measured at clinical stability when compared with the start of the pulmonary exacerbation (median values of $1.2 \times 10^{7} \mathrm{CFU} \cdot \mathrm{g}^{-1}$ and $1.0 \times 10^{7} \mathrm{CFU} \cdot \mathrm{g}^{-1}$ of sputum, respectively).

\section{Discussion}

This is the first study to test the hypothesis that QSSMs may be correlated with clinical status in the context of a pulmonary exacerbation in adults with CF and chronic pulmonary $P$. aeruginosa. We have observed for the first time that a number of different QSSMs are detectable in sputum, plasma and urine in this patient group at the start of a pulmonary exacerbation. In particular, one QSSM (NHQ) in the plasma was elevated at the start of a pulmonary exacerbation, when compared to the baseline, and

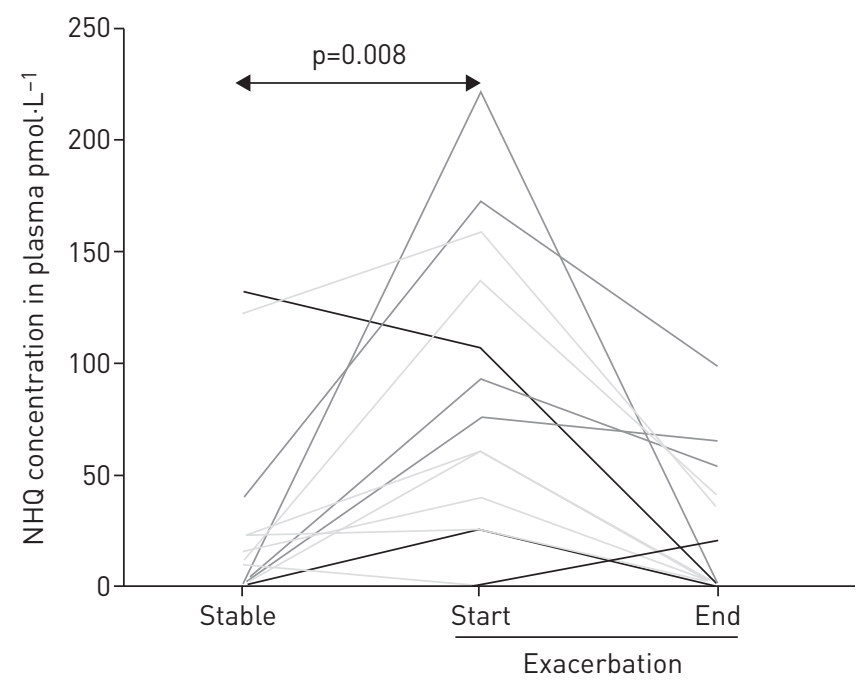

FIGURE 1 Plasma concentrations of 2-nonyl-4-hydroxy-quinoline (NHQ) in 29 patients with cystic fibrosis at clinical stability (eight samples before the exacerbation and 21 samples after the exacerbation of interest) compared to the beginning of a pulmonary exacerbation. 
decreased significantly after treatment, suggesting this molecule may be sensitive to changes in clinical status and has particular potential as a biomarker in this setting.

The strengths of this study include the prospective study design and testing of predetermined hypotheses. The cohort was well characterised with a broad range in lung disease severity, suggesting our findings may be applicable to wider populations with similar severity of respiratory disease, but not necessarily those with mild or end-stage disease. The samples were collected following a rigorous protocol and processed in line with conventional practice, thereby reducing any variance in the signals generated by random error. We excluded patients that had previously isolated other respiratory pathogens capable of producing alkyl quinolones, such as Burkholderia cepacia complex [16], used hospital microbiological culture to validate our findings, and quantitative microbiological measures of $P$. aeruginosa to allow more detailed statistical assessment of the associations of interest. This had the added advantage of allowing comparison of the new candidate biomarkers alongside conventional practice, demonstrating that a positive QSSM signal was present even when the conventional microbiological culture was negative for P. aeruginosa. The availability of measures of lung function and quantitative markers of pulmonary inflammation objectively confirmed the observed clinical recovery of the individual patients. Additional paired data from a period of clinical stability allowed more detailed longitudinal comparisons of QSSM concentrations with clinical status for approximately half of the participants.

Our study has some limitations that need consideration. The study design required the measurement of eight QSSMs using a targeted and quantitative LC-MS/MS method. This was a necessary requirement of this study design to permit the identification of the QSSMs, which have the greatest potential as biomarkers, and the testing of multiple hypotheses requires consideration when interpreting these data. The large number of negative values that we observed for all biofluids in patients known to be infected with $P$. aeruginosa raises the possibility that QSSMs are present but below the lower limit of quantification (LLOQ) (for further details refer to the online supplementary material) for detection by the LC-MS/MS method. Alternatively, the concurrent use of azithromycin in the majority of CF patients in this study may have resulted in a reduction in QSSM concentrations [17], or some populations of P. aeruginosa may have evolved with reduced or absent ability to produce QSSMs [18]. The collection of urine samples was not standardised and used a random "catch", which would be expected to increase the random error of any molecules measured as the concentration may vary. Future studies assessing the potential of urinary QSSMs as biomarkers should consider correcting for urinary creatinine concentration to allow for differential dilution. Future studies may also consider comparisons between QSSM levels and systemic biomarkers of inflammation, such as $\mathrm{C}$ reactive protein. Finally, we were unable to generate detailed phenotypes for the bacterial population isolated from the clinical samples due to resource constraints, and this is an option for future studies in this cohort.

There are several observations in our data that require further consideration. Firstly, the QSSMs that changed over the course of antibiotic treatment were found to decline in the plasma but not in the sputum samples collected. All samples were collected and analysed in the same rigorous manner and we doubt that this is an artefact and consider that it is likely to be a true negative. It is possible that concentrations of QSSMs in plasma may reflect the total QSSM load in the host more accurately than sputum samples from the lungs, which are susceptible to sampling variation as a consequence of the heterogeneous nature of infection with $P$. aeruginosa in the CF airways [19]. Differences in local environmental conditions within the lung, such as nutrient availability, may influence QSSM production [20] and may contribute to the relatively poor correlations between QSSM levels and the quantity of $P$. aeruginosa observed in this study and by others [21]. Alternatively, the clearance of QSSMs may differ between sputum and plasma and an interval of greater than a median of 14 days may be required to detect changes in these measures in the lung. QSSMs in the blood may be excreted or metabolised faster than those in the lung and, thus, would be expected to have a shorter half-life, a hypothesis that is consistent with our data. This has important implications for future studies that aim to monitor sputum and plasma QSSMs over time. The second observation that requires consideration is that some QSSMs were positively correlated with FEV1 in cross-sectional analysis at the start of a pulmonary exacerbation and the reasons for this unexpected finding are not known. One possible explanation is that patients with lower lung function are likely to have had a higher cumulative exposure to antibiotics that could potentially reduce QSSM production by $P$. aeruginosa $[22,23]$. Alternatively, patients with lower lung function may have a longer duration of infection with $P$. aeruginosa and this may be associated with a reduction in QSSM levels as a result of cumulative mutations in the QS regulatory genes over time [24, 25].

Overall, our data suggest that clinical status may be associated with changes in systemic QSSMs, rather than absolute changes in bacterial numbers, and that i.v. antibiotics may lead to a reduction in systemic QSSMs in the context of a pulmonary exacerbation. One potential explanation for this observation is that some of the therapeutic effect of systemic antibiotics may be mediated via QSSMs. This hypothesis is consistent with studies 
that have shown that bacterial load is poorly correlated with clinical status $[26,27]$, that the CF microbiome is relatively resistant to antibiotics [28] and that several classes of antibiotics routinely used in the management of patients with CF may inhibit QSSM or QS-mediated virulence factor production [22, 23, 29, 30].

The implications of these data are that QSSMs have the potential to provide biomarkers for P. aeruginosa and this may lead to the possibility of non-pulmonary measures of infection using plasma or urinary samples. In particular, plasma biomarkers that are sensitive to i.v. antibiotics and subsequent clinical improvement may provide a new tool to assess response to treatment in future. We observed consistent signals in molecules from the alkyl quinolone category, which is compatible with the hypothesis that they have the greatest potential as novel biomarkers for $P$. aeruginosa as they are only produced by this bacterium and a few closely related species and $P$. aeruginosa is the only species known to produce PQS [16]. This study represents an early stage in the investigation of QSSMs as novel biomarkers for P. aeruginosa and to bring this test to routine clinical practice would require further validation steps and the development of simple point of care assay, similar to those used in diabetic control monitoring. These data also suggest that investigation into the potential use of QSSMs as biomarkers for the early detection of P. aeruginosa should be explored, given the current limitations of diagnosing lower respiratory tract infections in those unable to expectorate sputum. In future, this test may have wider clinical applications in other diseases in which $P$. aeruginosa is an important pathogen, such as non-CF bronchiectasis [31] and chronic obstructive pulmonary disease [32].

In conclusion, we report for the first time that QSSMs are detectable in sputum, plasma and urine in patients with a pulmonary exacerbation of CF. In particular, plasma NHQ is increased at the presentation with an exacerbation compared to clinical stability, and decreases after administration of i.v. antibiotics, suggesting this molecule may be sensitive to clinical changes and has potential as a biomarker to assess response to treatment in future.

\section{Acknowledgements}

We would like to thank all the individuals who took part in this study for their help. The authors' contributions were as follows: Helen L. Barr contributed to the acquisition, analysis and interpretation of data, wrote the initial draft of the manuscript and approved the final version to be published. Nigel Halliday contributed to the acquisition, analysis and interpretation of data, drafting the manuscript and final approval of the final version to be published; Miguel Cámara, Paul Williams, David A. Barrett, Alan R. Smyth and Alan J. Knox contributed to the conception and design, acquisition and interpretation of data, critical revision of the manuscript and final approval of the version to be published; Rebecca Simms contributed to the acquisition and analysis of data, critical revision of the manuscript and final approval of the version to be published; Douglas L. Forester contributed to the conception and design, acquisition and interpretation of data, drafting the manuscript and final approval of the version to be published; David Honeybourne, Edward F. Nash, Joanna L. Whitehouse, Andrew Clayton and Jane Dewar contributed to the acquisition of data, critical revision of the manuscript and final approval of the version to be published; and Push/pull contributed to the concept and design, analysis and interpretation of data, drafting the manuscript and final approval of the version to be published and is the guarantor.

\section{References}

1 Konstan MW, Morgan WJ, Butler SM, et al. Risk factors for rate of decline in forced expiratory volume in one second in children and adolescents with cystic fibrosis. J Pediatr 2007; 151: 134-139.

2 Emerson J, Rosenfeld M, McNamara S, et al. Pseudomonas aeruginosa and other predictors of mortality and morbidity in young children with cystic fibrosis. Pediatr Pulmonol 2002; 34: 91-100.

3 Williams P, Cámara M. Quorum sensing and environmental adaptation in Pseudomonas aeruginosa: a tale of regulatory networks and multifunctional signal molecules. Curr Opin Microbiol 2009; 12: 182-191.

4 Jaffar-Bandjee MC, Lazdunski A, Bally M, et al. Production of elastase, exotoxin A, and alkaline protease in sputa during pulmonary exacerbation of cystic fibrosis in patients chronically infected by Pseudomonas aeruginosa. J Clin Microbiol 1995; 33: 924-929.

5 Middleton B, Rodgers HC, Cámara $\mathrm{M}$, et al. Direct detection of $\mathrm{N}$-acylhomoserine lactones in cystic fibrosis sputum. FEMS Microbiol Lett 2002; 207: 1-7.

6 Struss AK, Nunes A, Waalen J, et al. Toward implementation of quorum sensing autoinducers as biomarkers for infectious disease states. Anal Chem 2013; 85: 3355-3362.

7 Smyth AR, Cifelli PM, Ortori CA, et al. Garlic as an inhibitor of Pseudomonas aeruginosa quorum sensing in cystic fibrosis-a pilot randomized controlled trial. Pediatr Pulmonol 2010; 45: 356-362.

8 Rosenfeld M, Emerson J, Williams-Warren J, et al. Defining a pulmonary exacerbation in cystic fibrosis. J Pediatr 2001; 139: 359-365.

9 Flume PA, Mogayzel PJ Jr, Robinson KA, et al. Cystic fibrosis pulmonary guidelines: treatment of pulmonary exacerbations. Am J Respir Crit Care Med 2009; 180: 802-808.

10 Miller MR, Hankinson J, Brusasco V, et al. Standardisation of spirometry. Eur Respir J 2005; 26: 319-338.

11 The UK Cystic Fibrosis Trust Diabetes Working Group. Cystic Fibrosis Our Focus: Management of Cystic Fibrosis Related Diabetes Mellitus. Bromley, Cystic Fibrosis Trust, 2004. Available from: http://cysticfibrosis.org.uk/media/ 82028/diabetes-mellitus-management-jun04.pdf

12 The UK Cystic Fibrosis Trust Microbiology Laboratory Standards Working Group. Laboratory Standards for Processing Mircobiological Samples from People with Cystic Fibrosis. Bromley, Cystic Fibrosis Trust, 2004. Available from: www.cysticfibrosis.org.uk/media/82034/CD_Laboratory_Standards_Sep_10.pdf

13 Pavord ID, Pizzichini MM, Pizzichini E, et al. The use of induced sputum to investigate airway inflammation. Thorax 1997; 52: 498-501. 
Ortori CA, Atkinson S, Chhabra SR, et al. Comprehensive profiling of $N$-acylhomoserine lactones produced by Yersinia pseudotuberculosis using liquid chromatography coupled to hybrid quadrupole-linear ion trap mass spectrometry. Anal Bioanal Chem 2007; 387: 497-511.

15 Ortori CA, Dubern JF, Chhabra SR, et al. Simultaneous quantitative profiling of $N$-acyl-L-homoserine lactone and 2-alkyl-4(1H)-quinolone families of quorum-sensing signaling molecules using LC-MS/MS. Anal Bioanal Chem 2011; 399: 839-850.

16 Diggle SP, Lumjiaktase P, Dipilato F, et al. Functional genetic analysis reveals a 2-Alkyl-4-quinolone signaling system in the human pathogen Burkholderia pseudomallei and related bacteria. Chem Biol 2006; 13: 701-710.

17 Hoffmann N, Lee B, Hentzer M, et al. Azithromycin blocks quorum sensing and alginate polymer formation and increases the sensitivity to serum and stationary-growth-phase killing of Pseudomonas aeruginosa and attenuates chronic $P$. aeruginosa lung infection in $\mathrm{Cftr}^{-/-}$mice. Antimicrob Agents Chemother 2007; 51: 3677-3687.

18 Diggle SP, Griffin AS, Campbell GS, et al. Cooperation and conflict in quorum-sensing bacterial populations. Nature 2007; 450: 411-414.

19 Meyer KC, Sharma A. Regional variability of lung inflammation in cystic fibrosis. Am J Respir Crit Care Med 1997; 156: 1536-1540.

20 Palmer KL, Mashburn LM, Singh PK, et al. Cystic fibrosis sputum supports growth and cues key aspects of Pseudomonas aeruginosa physiology. J Bacteriol 2005; 187: 5267-5277.

21 Wilder CN, Allada G, Schuster M. Instantaneous within-patient diversity of Pseudomonas aeruginosa quorum-sensing populations from cystic fibrosis lung infections. Infect Immun 2009; 77: 5631-5639.

22 Garske LA, Beatson SA, Leech AJ, et al. Sub-inhibitory concentrations of ceftazidime and tobramycin reduce the quorum sensing signals of Pseudomonas aeruginosa. Pathology 2004; 36: 571-575.

23 Tateda K, Comte R, Pechere JC, et al. Azithromycin inhibits quorum sensing in Pseudomonas aeruginosa. Antimicrob Agents Chemother 2001; 45: 1930-1933.

24 Bjarnsholt $\mathrm{T}$, Jensen $\mathrm{P} \emptyset$, Jakobsen TH, et al. Quorum sensing and virulence of Pseudomonas aeruginosa during lung infection of cystic fibrosis patients. PLoS One 2010; 5: e10115.

25 Smith EE, Buckley DG, Wu Z, et al. Genetic adaptation by Pseudomonas aeruginosa to the airways of cystic fibrosis patients. Proc Natl Acad Sci USA 2006; 103: 8487-8492.

26 Okusanya OO, Bhavnani SM, Hammel J, et al. Pharmacokinetic and pharmacodynamic evaluation of liposomal amikacin for inhalation in cystic fibrosis patients with chronic pseudomonal infection. Antimicrob Agents Chemother 2009; 53: 3847-3854.

27 Mowat E, Paterson S, Fothergill JL, et al. Pseudomonas aeruginosa population diversity and turnover in cystic fibrosis chronic infections. Am J Respir Crit Care Med 2011; 183: 1674-1679.

28 Fodor AA, Klem ER, Gilpin DF, et al. The adult cystic fibrosis airway microbiota is stable over time and infection type, and highly resilient to antibiotic treatment of exacerbations. PLoS One 2012; 7: e45001.

29 Nalca Y, Jänsch L, Bredenbruch F, et al. Quorum-sensing antagonistic activities of azithromycin in Pseudomonas aeruginosa PAO1: a global approach. Antimicrob Agents Chemother 2006; 50: 1680-1688.

30 Skindersoe ME, Alhede M, Phipps R, et al. Effects of antibiotics on quorum sensing in Pseudomonas aeruginosa. Antimicrob Agents Chemother 2008; 52: 3648-3663.

31 Goeminne P, Dupont L. Non-cystic fibrosis bronchiectasis: diagnosis and management in 21st century. Postgrad Med J 2010; 86: 493-501.

32 Murphy TF, Brauer AL, Eschberger K, et al. Pseudomonas aeruginosa in chronic obstructive pulmonary disease. Am J Respir Crit Care Med 2008; 177: 853-860. 